\title{
Adaptation of silage maize varieties under extreme northern growing condions in Finland
}

\author{
SePpo Pulli \\ University of Helsinki, Dept. of Plant Husbandry, 00710 Helsinki 71 \\ P. M. A. TigerstedT \\ University of Helsinki, Dept. of Plant Breeding, 00710 Helsinki 71 \\ Osmo Kara \\ University of Helsinki, Dept. of Farm Engineering, 00710 Helsinki 71 \\ G. BRÜNINGHAUS \\ Wiurila, 24910 Halikko
}

\begin{abstract}
Trials with maize varieties from various places in the world were started in 1975. In preliminary trials in 1975, 280 varieties were tested. Between 19 and 23 varieties were selected for ordinary variety tests in $1976-78$ at the University farm in Siuntio.

Weather conditions, particularly average daily temperatures in 1975 were better than the long term averages, and in $1976-78$ far below the average growing conditions.

Dry matter yields of the seven harvested silage varieties in 1975 varied between 5.8 and 11.5 tons/ha. In $1976-78$ the variation in DM yields was $3.8-8.0$ tons/ha among $19-23$ varieties. In 1975,44 varieties out of 280 produced mature seed. Only one variety matured in 1978 , but none in 1976-77. The developmental stage of silage maize is primarily determined by ear percentage and secondarily by DM \%. In 1975 the average ear \% of seven varieties was $49.1 \%$, in $197818.1 \%$ and in $1976-77$ only $4.0-5.7 \%$ in DM.

As a result of the variety tests promising varieties from Yugoslavia, France and Germany could be found. It can be concluded from the long term temperature data that with very early hybrid varieties a mature grain yield can be harvested twice in ten years. Good quality silage material can be harvested six times in ten years and a satisfactory crop can be obtained eight times in then years.

The limiting factor for the growth and development of maize in Finland is the low average temperature of the growing season. Important but less significant is the length of the vegetative period, which is determined by the first killing frost in the fall. The temperature deficit is particularly critical at the beginning of the growing season.
\end{abstract}

\section{Introduction}

The adaptability of maize varieties to Finnish climatic conditions was first studied in the 1930 's. The most important results were published by A. I. Virtanen. According to Virtanen the fresh weight of the best maize variety 
varied between $64-107$ tons/ha, the average yield being 72.5 tons/ha (VIRTANEN 1938). At this time silage maize was widely cultivated on private farms. VIRTANEN's data, obtained from 131 farms in 1937, summarizes the averagc fresh yield of maize at 41 tons/ha (VIRTANEN 1938).

At the Plant Breeding Institute of Hankkija in Tammisto maize varieties have been studied since the early 1930's. In 1953-54 the fresh yield of silage maize varieties varied between $36-80$ tons/ha. The average fresh weight of varieties was 54 tons/ha. The dry matter percentage (DM \%) was about 11.7-13.6 and the harvested yield about 7.5 tons DM/ha. In 1955 in Tammisto maize suffered from drought and the fresh yields reached only a level of $9-22$ tons/ha. In 1957-58 in Tammisto the yields were between $46-83$ tons/ha (RAvantTI 1956, 1960). In later research program the average dry matter yields of silage maize varieties varied between $5.4-6.8$ tons/ha in $1965-69$ and between 5.3-10.6 tons/ha in 1973-74 (RAIninko 1970, JuUti and RAININKo 1975).

At the Agricultural Research Center in Tikkurila maize trials were carried out already in the 1930's but were interrupted during the war. In 1950 they were resumed in connection with the FAO hybrid maize program. These trials were mainly carried out by the Dept. of Plant Husbandry in Tikkurila and three experimental stations in southern Finland. Yields varied widely in various years and were generally low (YLLÖ 1962). In 1937 the variety tests showed a yield variation of $17.7-54.1$ tons/ha the average yield of all varieties being 31.2 tons/ha. In $1950,1952-53,1956$ and 1958 the trials failed more or less completely mainly because of cool growing seasons, early autumn frosts and the use of largely nonadapted varieties.

Maize variety trials in the 1950's and in the early 1960's showed that maize was an uncertain field crop under Finnish conditions. However, it was concluded that it can be grown with certain limitations in South Finland, principally on fertile, warm soils, where there is little danger of autumn frosts (YLLÖ 1962).

As a result of favorable development of hybrid maize breeding in Central Europe during the last ten years, the economic northern limit of maize growing has been moved to northern Germany and Denmark. Due to this development a new start in maize research was made in 1975 in Finland. The new survey of maize production in Finland began with the testing of large collections of breeding material from all over the world. Subsequently this material has been tested under different cultivation conditions. The research was financed by the Cultural Foundation of Finland in 1975-77 and the Ford-Foundation in 1978 .

\section{Materials and methods}

The preliminary variety tests were established on the Wiurila farm in Halikko near Salo in 1975. A collection of 280 varieties was tested in one replication. This screening nursery served the purpose of selecting promising material from a world wide collection of potential maize lines and varieties. 
In subsequent years $(1976-78)$ the experimental desing was a randomized block with 4 replications. This was carried out on the Suitia Helsinki University farm:

\begin{tabular}{|c|c|c|c|c|}
\hline & Screening nursery & & Variety tria & \\
\hline & 1975 & 1976 & 1977 & 1978 \\
\hline 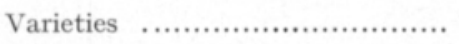 & 280 & 19 & 20 & 23 \\
\hline 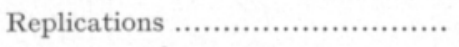 & 1 & 4 & 4 & 4 \\
\hline 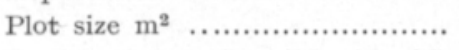 & 16 & 48 & 26 & 16 \\
\hline Fertilization $\mathrm{kg} / \mathrm{ha} \quad(15-15-15)$ & 1000 & 1000 & 1000 & 1000 \\
\hline Seeding date ............................ & May 5 & May 17 & May 12 & May 17 \\
\hline 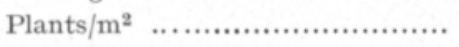 & 10 & 10 & 10 & 10 \\
\hline Row spacing $\mathrm{cm}$........................ & 80 & 80 & 80 & 80 \\
\hline Plant spacing $\mathrm{cm}$........................ & 12.5 & 12.5 & 12.5 & 12.5 \\
\hline Seeding depth $\mathrm{cm} . \ldots \ldots \ldots \ldots \ldots \ldots \ldots$ & 7 & 7 & 7 & 7 \\
\hline 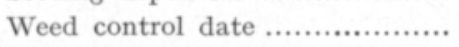 & May 20 & June 2 & May 20 & May 23 \\
\hline 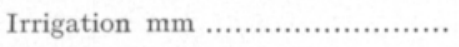 & $2 \times 25$ & $2 \times 25$ & 25 & 25 \\
\hline Harvest date ............................ & Oct. 27 & Oct. 6 & Sept. 24 & Sept. 23 \\
\hline
\end{tabular}

A two-unit drill (Kleine Maxicorn) was used as a seeding unit. Fertilizer was applied to both sides and below the seed. The plots were pre-emergence weed-controlled with Gesaprim-50, 3-4.5 1/ha. Insects were controlled with Roxion $0.81 /$ ha. The yield data consisted of the fresh weight, dry matter $\%$, and protein content $(\%)$ in dry matter. Height measurements were taken once a week during the growing season.

\section{Weather conditions}

Temperature conditions during the growing seasons $1976-78$ were generally below long-term averages for $1931-60$ (Table 1). The year 1975 represents an above normal growing season, which resulted in a well matured high grain yield. Especially the warm and long autumn in 1975 boosted the growth and development of the maize plant.

During the whole research period the precipitation stayed below the long term average (Table 1). The maize was irrigated every spring after emergence and a second time before flowering in 1975-76.

During the growing seasons 1975-78 the last harmful frosts occurred during the time of May 29 - June 18 (Table 1). These night frosts were harmful for the growth but did not kill the crop. The autumn frosts $\left(-2^{\circ} \mathrm{C}\right)$ in 1976-77 stopped the growth of maize in early September.

The growing seasons $1975-78$ represent $170-130$ growing days from the seeding date to the harvest (Table 2). Calculated degree days show the levels between $2237^{\circ} \mathrm{C}$ and $1667^{\circ} \mathrm{C}$. If expressed in effective degree days for maize $\left(\mathrm{t}^{\circ}-10^{\circ} \mathrm{C}\right)$ the levels are between $688^{\circ} \mathrm{C}$ and $412^{\circ} \mathrm{C}$. Calculated corn heat units (CHU) as shown by Brown (1975) $2259^{\circ} \mathrm{C}$ and $1625^{\circ} \mathrm{C}$ do not differ very much from the degree days. 
Table 1. Weather conditions in the experimental area in 1975-78.

\begin{tabular}{|c|c|c|c|c|c|c|}
\hline \multirow{2}{*}{\multicolumn{2}{|c|}{ Period }} & \multicolumn{4}{|c|}{ Years } & \multirow{2}{*}{$\begin{array}{l}\text { Avg. } \\
1930-60\end{array}$} \\
\hline & & 1975 & 1976 & 1977 & 1978 & \\
\hline \multicolumn{7}{|c|}{ Avg. daily temperature ${ }^{\circ} \mathrm{C}$} \\
\hline May $1-30 \ldots \ldots$. & (n).............. & 11.6 & 10.2 & 9.4 & 9.3 & 9.0 \\
\hline May $30-$ June 29 & ................ & 12.9 & 12.6 & 13.8 & 14.2 & 14.2 \\
\hline June $30-$ July 29 & ................ & 17.0 & 15.0 & 14.3 & 14.5 & 16.8 \\
\hline July 30 - Aug. 28 & ................ & 16.7 & 13.5 & 13.8 & 13.6 & 14.9 \\
\hline \multirow[t]{2}{*}{ Aug. $29-$ Sept. 27} & ................. & 13.6 & 8.7 & 8.2 & 8.8 & 9.9 \\
\hline & Avg. & 14.4 & 12.0 & 11.9 & 12.1 & 13.0 \\
\hline \multicolumn{7}{|c|}{ Precipitation $\mathrm{mm}$} \\
\hline May $1-30 \ldots \ldots$. & (n................ & 40 & 14 & 16 & 6 & 39 \\
\hline May 31 - June 29 & ................. & 29 & 51 & 40 & 52 & 46 \\
\hline June $30-$ July 29 & ................. & 38 & 40 & 94 & 70 & 73 \\
\hline July $30-$ Aug. 28 & ................ & 40 & 58 & 58 & 82 & 75 \\
\hline \multirow[t]{2}{*}{ Aug. $29-$ Sept. 27} & ................. & 60 & 68 & 64 & 75 & 65 \\
\hline & Total & 207 & 231 & 272 & 285 & 298 \\
\hline \multicolumn{7}{|c|}{ Killing frost } \\
\hline \multirow{2}{*}{\multicolumn{2}{|c|}{ 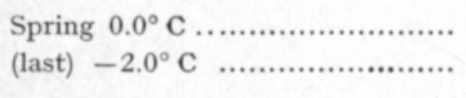 }} & $3 / 6$ & $10 / 6$ & $2 / 6$ & $18 / 6$ & \\
\hline & & $30 / 5$ & $29 / 5$ & $1 / 6$ & $18 / 6$ & \\
\hline \multirow{2}{*}{\multicolumn{2}{|c|}{ 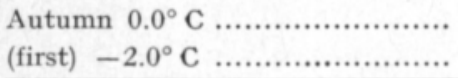 }} & $9 / 10$ & $22 / 8$ & $19 / 8$ & $14 / 8$ & \\
\hline & & $10 / 10$ & $8 / 9$ & $9 / 9$ & $21 / 9$ & \\
\hline
\end{tabular}

Table 2. Growing days, degree days (DD), effective degree days (EDD) and corn heat units $(\mathrm{CHU})$ of growing seasons $1975-78$ in southern Finland.

\begin{tabular}{|c|c|c|c|c|c|}
\hline & \multicolumn{5}{|c|}{ Year } \\
\hline & 1975 & 1976 & 1977 & 1978 & Avg. \\
\hline 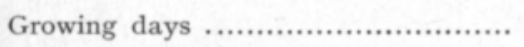 & 170 & 143 & 136 & 130 & 145 \\
\hline Degree days (DD) ${ }^{\circ} \mathrm{C} . \ldots \ldots \ldots \ldots \ldots \ldots . . . . . .$. & 2237 & 1667 & 1679 & 1714 & 1824 \\
\hline Effective degree days (EDD) ${ }^{\circ} \mathrm{C} \ldots \ldots$. & 688 & 412 & 433 & 455 & 497 \\
\hline Corn heat units $(\mathrm{CHU}){ }^{\circ} \mathrm{C}$............. & 2259 & 1656 & 1625 & 1699 & 1810 \\
\hline
\end{tabular}

$\mathrm{DD}=\Sigma\left(\right.$ Avg daily $\left.{ }^{\circ} \mathrm{C}\right)$

$\mathrm{EDD}=\Sigma\left(\right.$ Avg daily $\left.{ }^{\circ} \mathrm{C}-10^{\circ} \mathrm{C}\right)$

$\left.\mathrm{CHU}=\Sigma(\text { Daily } \mathrm{Y} \max +\text { daily } \mathrm{Y} \min )^{1}\right)$

2

1) $\mathrm{Y} \max =3.33(\mathrm{~T} \max -10)-0.084(\mathrm{~T} \max 10)^{2}$

$\mathrm{Y} \min =1.8 \quad(\mathrm{~T} \min -4.44)$

\section{Results and discussion}

\section{Yields}

Under temperature conditions above average, as in 1975, 44 of the studied 280 varieties produced a matured grain. The seven best varieties showed approximate yield levels of 5.8-11.5 tons total dry matter (DM)/ha (Table 3). Total protein yields varied between 580 and $940 \mathrm{~kg} / \mathrm{ha}$. The grain yields of 
the seven varieties varied between $3279 \mathrm{~kg} / \mathrm{ha}$ and $5578 \mathrm{~kg} / \mathrm{ha}$. The total DM yields obtained in 1975 in Wiurila are similar to the Danishr esults (PEDERSEN 1975).

Table 3. Total yields, matured seed yields and dry matter content, protein content in DM and the amount of cars in the total yield in 1975 in southern Finland.

\begin{tabular}{|c|c|c|c|c|c|c|c|}
\hline \multirow{2}{*}{ Variety } & \multicolumn{3}{|c|}{ Total yield tons/ha } & \multirow{2}{*}{$\frac{\text { Matured }}{\text { Grain kg/ha }}$} & \multicolumn{3}{|c|}{ Content $\%$} \\
\hline & Fresh & DM & Prot. & & DM & Prot. & Ears \\
\hline LG 11 ........... & 44.9 & 11.4 & .86 & 5578 & 25.5 & 7.6 & 53.6 \\
\hline$L G 5 \ldots \ldots \ldots \ldots$ & 41.9 & 11.5 & .83 & 5550 & 27.4 & 7.3 & 52.5 \\
\hline LG $672508 \ldots \ldots$. & 39.5 & 11.0 & .94 & 4955 & 28.0 & 8.6 & 43.1 \\
\hline LG $775007 \ldots \ldots$ & 38.0 & 10.0 & .89 & 4828 & 26.4 & 8.9 & 46.0 \\
\hline$L G \quad 7 \ldots \ldots \ldots \ldots$ & 37.5 & 10.2 & .76 & 4659 & 27.2 & 7.5 & 50.2 \\
\hline$C P \quad 170 \ldots \ldots \ldots \ldots$ & 37.2 & 8.7 & .76 & 3930 & 23.3 & 8.8 & - \\
\hline Flash ................ & 22.0 & 5.8 & .58 & 3279 & 26.3 & 10.0 & - \\
\hline Avg. & 37.3 & 9.8 & .80 & 4683 & 26.3 & 8.4 & 49.1 \\
\hline
\end{tabular}

In 1976 under clearly unfavorable growing conditions the average total fresh and DM yields of 19 varieties were 34 tons/ha and 5.7 tons/ha (Table 4). The variation in fresh yields of all varieties was $23.8-44.8$ tons/ha and in dry matter yields $3.9-7.4$ tons/ha. Protein yields of all varieties averaged 630 $\mathrm{kg} / \mathrm{ha}$.

Table 4. Fresh weight, dry matter (DM) and protein yields and DM, protein and ear content $(\%)$ of silage corn varieties in southern Finland in 1976.

\begin{tabular}{|c|c|c|c|c|c|c|}
\hline \multirow{2}{*}{ Variety } & \multicolumn{3}{|c|}{ Yields tons/ha } & \multicolumn{3}{|c|}{ Content \% } \\
\hline & Fresh wt & DM & Prot. & DM & Prot. & Ears \\
\hline LG 11 .................... & $44.8^{\mathrm{e}}$ & $7.4^{\mathrm{e}}$ & $.8 \mathrm{i}^{\mathrm{c}}$ & $16.6^{\mathrm{ab}}$ & 10.9 & 4.0 \\
\hline 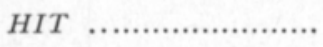 & $40.3^{\mathrm{be}}$ & $6.9^{\mathrm{be}}$ & $.75^{\mathrm{be}}$ & $17.1^{\mathrm{ab}}$ & 10.9 & 7.0 \\
\hline 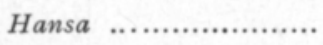 & $39.1^{\mathrm{be}}$ & $6.7^{\mathrm{be}}$ & $.75^{\mathrm{be}}$ & $17.0^{\mathrm{ab}}$ & 11.3 & 8.7 \\
\hline 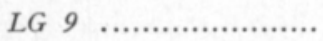 & $38.2^{\mathrm{be}}$ & $6.6^{\mathrm{be}}$ & $.74^{\mathrm{be}}$ & $17.4^{\mathrm{ab}}$ & 11.2 & 3.0 \\
\hline 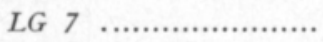 & $38.3^{\mathrm{be}}$ & $6.3 \mathrm{bc}$ & $.68^{\mathrm{be}}$ & $16.3^{\mathrm{ab}}$ & 10.8 & 6.7 \\
\hline 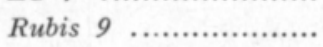 & $35.8^{\mathrm{b}}$ & $6.2^{\mathrm{bc}}$ & $.64^{\mathrm{bc}}$ & $17.6^{\mathrm{ab}}$ & 10.3 & 3.6 \\
\hline 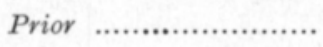 & $40.2^{\text {be }}$ & $6.1^{\mathrm{be}}$ & $.69 \mathrm{be}$ & $15.2^{\mathrm{a}}$ & 11.4 & 2.1 \\
\hline CP $170 \ldots \ldots \ldots \ldots \ldots \ldots \ldots$ & $33.3 \mathrm{~b}$ & $6.0^{\mathrm{be}}$ & $.68^{\text {be }}$ & $18.0^{\mathrm{b}}$ & 11.4 & 3.3 \\
\hline 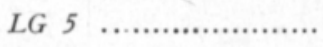 & $33.8^{\mathrm{b}}$ & $5.8^{\mathrm{be}}$ & $.66^{\mathrm{be}}$ & $17.2^{\mathrm{ab}}$ & 11.4 & 2.6 \\
\hline ACG 200 .................. & $36.9^{\mathrm{be}}$ & $5.8^{\mathrm{b}}$ & $.65^{\mathrm{bc}}$ & $15.7^{\mathrm{ab}}$ & 11.3 & 3.2 \\
\hline Zelder $75-102 \ldots \ldots \ldots$ & $33.6^{\mathrm{b}}$ & $5.6^{\mathrm{b}}$ & $.62^{\mathrm{b}}$ & $17.0^{\mathrm{ab}}$ & 11.0 & 7.6 \\
\hline 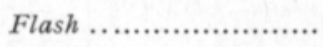 & $34.3^{\mathrm{b}}$ & $5.6^{\mathrm{b}}$ & $.61^{\mathrm{ab}}$ & $16.4^{\mathrm{ab}}$ & 10.9 & 6.0 \\
\hline 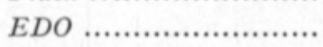 & $30.6^{\mathrm{ab}}$ & $5.4^{\mathrm{ab}}$ & $.64^{\mathrm{be}}$ & $17.4^{\mathrm{ab}}$ & 11.9 & 8.8 \\
\hline 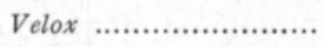 & $33.4^{\mathrm{b}}$ & $5.3^{\mathrm{ab}}$ & $.56^{\mathrm{ab}}$ & $15.9^{\mathrm{ab}}$ & 10.6 & 3.8 \\
\hline ACG $188 \ldots \ldots \ldots \ldots \ldots . . . . .$. & $32.5^{b}$ & $5.0^{\mathrm{ab}}$ & $.60^{\mathrm{ab}}$ & $15.6^{\mathrm{ab}}$ & 12.0 & 3.2 \\
\hline Jacques Cartieur ......... & $27.5^{\mathrm{ab}}$ & $4.7 \mathrm{ab}$ & $.54 \mathrm{ab}$ & $17.4^{\mathrm{ab}}$ & 11.5 & 4.2 \\
\hline Zelder $75-104 \ldots \ldots \ldots$ & $25.9^{\mathrm{ab}}$ & $4.4^{\mathrm{ab}}$ & $.44^{\mathrm{a}}$ & $17.0^{\mathrm{ab}}$ & 9.9 & 2.4 \\
\hline ETA-IPHO $182 \ldots \ldots .$. & $23.2^{\mathrm{a}}$ & $4.2^{\mathrm{ab}}$ & $.48^{\mathrm{ab}}$ & $18.6^{b}$ & 11.3 & 2.9 \\
\hline \multirow[t]{2}{*}{ Zelder $75-103 \ldots \ldots \ldots$} & $23.8^{\mathrm{a}}$ & $3.9 \mathrm{a}$ & $.49^{\mathrm{ab}}$ & $16.4^{\mathrm{ab}}$ & 12.6 & 6.1 \\
\hline & 34.0 & 5.7 & .63 & 16.8 & 11.2 & 4.8 \\
\hline
\end{tabular}

LSD .05

8.4

1.6

.17 
The fresh yields, dry matter yields and protein yields in 1977 equalled the ones obtained in 1976, although the growing conditions were even poorer than those in 1976 (Table 5). In 1978 (Table 6) the average yield of 23 varieties was $43.3(35.4-50.9)$ tons/ha fresh yield, $6.6(4.9-8.0)$ tons/ha DM yield and $430(340-500) \mathrm{kg} / \mathrm{ha}$ protein yield. The yields of silage maize on 1976-78 equal those obtained by Andersson and LööF (1959) in Sweden.

\section{Quality of the yield}

The percentage of ears of the total dry matter yield is a good indicator of the growing factors available to maize in any growing season. In a very favorable years in 1975 the percentage of ears of all varieties was $49.1 \%$, in $197818.1 \%$ and in the very unfavorable years $1976-77$ only $4.0-5.7 \%$. The same trend, although not so clear, can be found in the dry matter content of the yield. The season 1975 represents a level of $23.3 \%$ and the years $1976-$ 78 a level of $15.1-16.8 \%$ (Tables $3,4,5,6$ ). The protein content of all varieties over all years is between $4.7 \%$ and $12.7 \%$. The figures equal those obtained in Sweden (NoRDFELT 1959).

\section{Varieties}

The varieties common to yield trials of the years $1976-78$ were $L G 5$, $L G$ 7, LG 9, LG 11, CP 170, Rubis 9, Flash and AGG 200 from France, Edo

Table 5. Fresh weight, dry matter (DM) and protein yields and DM, protein and ear content (\%) of silage corn varieties in southern Finland in 1977.

\begin{tabular}{|c|c|c|c|c|c|c|}
\hline \multirow{2}{*}{ Variety } & \multicolumn{3}{|c|}{ Yields tons/ha } & \multicolumn{3}{|c|}{ Content $\%$} \\
\hline & Fresh wt & DM & Prot. & DM & Prot. & Ears \\
\hline 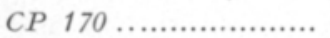 & $45.2^{\mathrm{e}}$ & $6.90^{\mathrm{e}}$ & $.72^{\mathrm{b}}$ & $15.3 \mathrm{ab}$ & 10.5 & 4.1 \\
\hline Prior $\ldots . . . . . . . . . . . . . . . . . . . .$. & $46.3^{\mathrm{e}}$ & $6.79^{e}$ & $.75^{b}$ & $14.6^{\mathrm{ab}}$ & 11.0 & 3.4 \\
\hline 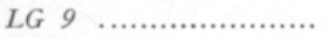 & $40.2^{\text {be }}$ & 6.21 be & $.65^{b}$ & $15.5^{\mathrm{ab}}$ & 10.4 & 2.0 \\
\hline$L G 7 \ldots \ldots \ldots \ldots \ldots \ldots \ldots$ & $40.7^{\text {be }}$ & $6.13^{\mathrm{be}}$ & $.61^{\mathrm{b}}$ & $15.1 \mathrm{ab}$ & 10.0 & 5.1 \\
\hline Rubis 9 .................. & $36.2^{\mathrm{b}}$ & $6.11^{\mathrm{be}}$ & $.66^{\mathrm{b}}$ & $16.9^{\mathrm{be}}$ & 10.8 & 7.1 \\
\hline Blizzard .................. & $41.5^{\mathrm{bc}}$ & $5.94^{\mathrm{be}}$ & $.69^{b}$ & $14.2^{\mathrm{a}}$ & 11.5 & 5.5 \\
\hline ACG $200 \ldots \ldots \ldots \ldots \ldots \ldots$ & 41.7 be & $5.94^{\mathrm{be}}$ & $.65^{b}$ & $14.2^{\mathrm{a}}$ & 11.0 & 4.8 \\
\hline Flash ...................... & $37.2^{\mathrm{b}}$ & $5.84^{\text {be }}$ & $.58^{\mathrm{b}}$ & $15.7 \mathrm{ab}$ & 10.0 & 6.4 \\
\hline$L G 11 \quad \ldots$ & $39.6^{\text {be }}$ & $5.82^{\text {be }}$ & $.59^{\mathrm{b}}$ & $14.9 \mathrm{ab}$ & 10.2 & 3.2 \\
\hline Velox $\cdot \ldots \ldots \ldots \ldots \ldots \ldots \ldots$ & $38.9^{\mathrm{be}}$ & $5.72^{\text {be }}$ & $.64^{\mathrm{b}}$ & $14.7 \mathrm{ab}$ & 11.2 & 6.1 \\
\hline$L G 5 \ldots$ & $37.8^{\mathrm{be}}$ & $5.68^{\text {be }}$ & $.61^{\mathrm{b}}$ & $15.1 \mathrm{ab}$ & 10.7 & 5.0 \\
\hline$H I T \ldots$. & $36.0^{\mathrm{b}}$ & $5.39 \mathrm{~b}$ & $.61^{\mathrm{b}}$ & $15.0^{\mathrm{ab}}$ & 11.5 & 6.4 \\
\hline Hansa .............. & $28.7 \mathrm{ab}$ & $5.04^{\mathrm{ab}}$ & $.54 \mathrm{ab}$ & $17.5^{\mathrm{e}}$ & 10.8 & 5.7 \\
\hline EDO ............ & $30.9 \mathrm{ab}$ & $5.01 \mathrm{ab}$ & $.52^{\mathrm{ab}}$ & $16.1^{\text {be }}$ & 10.4 & 8.9 \\
\hline Nibak .............. & $30.1 \mathrm{ab}$ & $4.81 \mathrm{ab}$ & $.55^{\mathrm{ab}}$ & $16.0^{\mathrm{b}}$ & 11.5 & 8.6 \\
\hline Kentala ............. & $31.4^{\mathrm{ab}}$ & $4.60^{\mathrm{ab}}$ & $.58^{\mathrm{ab}}$ & $14.7 \mathrm{ab}$ & 12.7 & 9.5 \\
\hline ACG $188 \ldots \ldots \ldots \ldots \ldots . . . .$. & $28.4^{\mathrm{a}}$ & $4.35^{\mathrm{ab}}$ & $.51 \mathrm{ab}$ & $15.1 \mathrm{ab}$ & 11.6 & 3.6 \\
\hline$M L \quad 06 \ldots \ldots \ldots \ldots \ldots \ldots$ & $27.7^{a}$ & $4.03^{\mathrm{a}}$ & $.46^{\mathrm{a}}$ & $14.5^{\mathrm{a}}$ & 11.4 & 8.3 \\
\hline ETA-I pho 182 ......... & $25.5^{\mathrm{a}}$ & $4.00^{\mathrm{a}}$ & $.45^{\mathrm{a}}$ & $15.1^{\mathrm{ab}}$ & 11.3 & 4.6 \\
\hline \multirow[t]{2}{*}{ Jacques Cartieur ......... } & $26.4^{\mathrm{a}}$ & $3.77 \mathrm{a}$ & $.44^{\mathrm{a}}$ & $14.4^{\mathrm{a}}$ & 11.6 & 5.3 \\
\hline & 35.5 & 5.40 & .59 & 15.2 & 11.0 & 5.7 \\
\hline
\end{tabular}

LSD .05

7.4

1.3

.14

1.4 
Table 6. Fresh weight, dry mater (DM) and protein yields and DM, protein and ear content (\%) of silage corn varieties in southern Finland in 1978.

\begin{tabular}{|c|c|c|c|c|c|c|}
\hline \multirow{2}{*}{ Variety } & \multicolumn{3}{|c|}{ Yields tons/ha } & \multicolumn{3}{|c|}{ Content $\%$} \\
\hline & Fresh wt & DM & Prot. & DM & Prot. & Ears \\
\hline ACG 201 ................. & $50.9 \mathrm{ab}$ & $8.04^{\mathrm{e}}$ & $.38 \mathrm{ab}$ & $15.8^{\mathrm{bc}}$ & 4.7 & 12.9 \\
\hline Forla ..................... & $47.4^{\mathrm{ab}}$ & $7.78^{\text {be }}$ & $.49 \mathrm{bc}$ & $16.4^{\mathrm{c}}$ & 6.3 & 25.0 \\
\hline$L G \quad 11 \quad \ldots \ldots \ldots \ldots \ldots \ldots \ldots$ & $47.2^{\mathrm{ab}}$ & $7.51^{\mathrm{bc}}$ & $.50^{\mathrm{be}}$ & $15.9^{\mathrm{bc}}$ & 6.7 & 8.4 \\
\hline CP $170 \ldots \ldots \ldots \ldots \ldots \ldots$ & $48.5^{\mathrm{ab}}$ & $7.28^{\mathrm{bc}}$ & $.47 \mathrm{be}$ & $15.0^{\mathrm{b}}$ & 6.4 & 16.4 \\
\hline$A C G \quad 185 \ldots \ldots \ldots \ldots \ldots \ldots . . . . . . .$. & $47.6^{\mathrm{ab}}$ & $7.28^{\mathrm{bc}}$ & $.48^{\mathrm{be}}$ & $15.6^{\mathrm{be}}$ & 6.6 & 16.2 \\
\hline 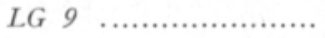 & $46.9 \mathrm{ab}$ & $7.15^{\text {be }}$ & $.47 \mathrm{bc}$ & $15.3^{\mathrm{be}}$ & 6.5 & 10.3 \\
\hline Rubis 9 .................. & $43.5^{\mathrm{ab}}$ & $6.86^{\text {be }}$ & $.38^{\mathrm{ab}}$ & 15.7 be & 5.6 & 19.3 \\
\hline ACG 189 .................. & $45.8^{\mathrm{ab}}$ & $6.77 \mathrm{be}$ & $.47^{\mathrm{bc}}$ & $14.8^{\mathrm{b}}$ & 6.9 & 19.3 \\
\hline$A C G \quad 167 \ldots \ldots \ldots \ldots \ldots \ldots . . . . . .$. & $45.8^{\mathrm{ab}}$ & $6.69 \mathrm{bc}$ & $.54^{\mathrm{e}}$ & $14.6^{\mathrm{ab}}$ & 8.1 & 19.9 \\
\hline Hansa ..................... & $41.7 \mathrm{ab}$ & $6.65^{\text {be }}$ & $.35 \mathrm{ab}$ & $16.8^{\mathrm{ed}}$ & 5.3 & 23.1 \\
\hline Blizzard .................... & $40.6^{\mathrm{ab}}$ & $6.58^{\mathrm{b}}$ & $.38^{\mathrm{ab}}$ & $14.6^{\mathrm{ab}}$ & 6.6 & 16.0 \\
\hline 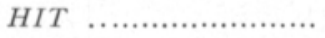 & $41.0^{\mathrm{ab}}$ & $6.51^{\mathrm{b}}$ & $.40^{\mathrm{ab}}$ & $15.9^{\mathrm{be}}$ & 6.2 & 26.4 \\
\hline 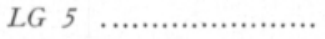 & $43.1 \mathrm{ab}$ & $6.50^{\mathrm{b}}$ & $.48^{\mathrm{bc}}$ & $15.1^{\mathrm{b}}$ & 7.4 & 13.9 \\
\hline 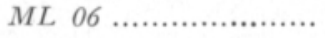 & $41.2^{\mathrm{ab}}$ & $6.22^{\mathrm{ab}}$ & $.44^{\mathrm{b}}$ & $15.1^{\mathrm{b}}$ & 7.0 & 23.5 \\
\hline 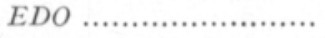 & $39.8^{\mathrm{ab}}$ & $6.20^{\mathrm{ab}}$ & $.39 \mathrm{ab}$ & $15.6^{\mathrm{be}}$ & 6.3 & 28.6 \\
\hline 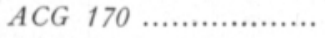 & $45.8^{\mathrm{ab}}$ & $6.20^{\mathrm{ab}}$ & $.48^{\mathrm{be}}$ & $13.6^{\mathrm{a}}$ & 7.8 & 10.8 \\
\hline Joran ..................... & $39.4 \mathrm{ab}$ & $6.14^{\mathrm{ab}}$ & $.49^{\mathrm{be}}$ & $15.6^{\mathrm{b}}$ & 8.0 & 16.6 \\
\hline Silac $233 \ldots \ldots \ldots \ldots \ldots$ & $42.7 \mathrm{ab}$ & $6.01 \mathrm{ab}$ & $.41 \mathrm{ab}$ & $14.0^{\mathrm{ab}}$ & 6.8 & 10.1 \\
\hline 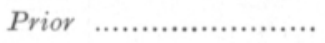 & $42.7 \mathrm{ab}$ & $6.00^{\mathrm{ab}}$ & $.37 \mathrm{ab}$ & $14.1 \mathrm{ab}$ & 6.1 & 16.9 \\
\hline Flash ...................... & $38.7 \mathrm{ab}$ & $5.96^{\mathrm{ab}}$ & $.36^{\mathrm{ab}}$ & $15.4^{\mathrm{be}}$ & 6.0 & 16.9 \\
\hline Mutin ....................... & $39.9 \mathrm{ab}$ & $5.86^{\mathrm{ab}}$ & $.44^{\mathrm{b}}$ & $14.7 \mathrm{ab}$ & 6.2 & 16.4 \\
\hline 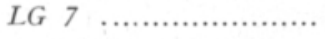 & $41.4 \mathrm{ab}$ & $5.81^{\mathrm{ab}}$ & $.44^{\mathrm{b}}$ & $14.0^{\mathrm{ab}}$ & 7.6 & 14.0 \\
\hline ACG $200 \ldots \ldots \ldots \ldots \ldots \ldots$ & $35.4^{\mathrm{a}}$ & $4.91^{\mathrm{a}}$ & $.34 \mathrm{a}$ & $13.9 \mathrm{ab}$ & 7.0 & 19.6 \\
\hline Avg. & 43.3 & 6.56 & .43 & 15.1 & 6.6 & 17.5 \\
\hline
\end{tabular}

Hit and Hansa from Germany and Prior, a Dutch variety. The yield level of these varied between $33.8-43.7$ tons/ha (Table 7). This yield level is the same as reported by VIRTANEN (1938) in private farming in the thirties. However, the varieties were less developed but the weather conditions much more favorable at that time.

In $1976-78$ the average dry matter yield of 12 varieties was 6.2 tons/ha. The nine best varieties did not differ statistically from each other. The yield level of $1976-78$ was 3.6 tons $\mathrm{DM} /$ ha lower than the yield level of seven varieties in 1975 revealing differences in growth conditions between the years. The average protein yield of $580 \mathrm{~kg} / \mathrm{ha}$ apparently very well represents the average protein producing ability of silage maize under Finnish growing conditions. The dry matter content of all varieties $(\mathbf{1 5 . 8} \%$ ) as well as the contribution of ears to the total DM $(9.3 \%)$ reveals the very low level of maturity.

All varieties ceased apical growth by the end of August. The average height of all varieties was $218 \mathrm{~cm}$. The protein content of the ear yield was higher than that of the stem (Table 8). As the dry mater yield of the ears was quite poor, the protein content of the ears plays an unimportant role in the total protein yield, especially in unfavorable seasons. 
Table 7. Average fresh, dry matter and protein yields tons/ha and percentage of DM, protein and ears in the total dry matter yield of twelve corn varieties studied in 1976-78 in Suitia.

\begin{tabular}{|c|c|c|c|c|c|c|c|}
\hline \multirow{2}{*}{ Variety } & \multicolumn{3}{|c|}{ Yields tons/ha } & \multirow{2}{*}{$\frac{\mathrm{DM}}{\%}$} & \multirow{2}{*}{$\begin{array}{c}\text { Prot. } \\
\% \\
\end{array}$} & \multirow{2}{*}{$\begin{array}{c}\text { Ears } \\
\% \\
\end{array}$} & \multirow{2}{*}{ Height } \\
\hline & Fresh & DM & Prot. & & & & \\
\hline$L G 11 \quad \ldots \ldots \ldots$ & $43.7^{b}$ & $6.93^{b}$ & $.64^{b}$ & $15.8^{b}$ & $9.3^{\mathrm{ab}}$ & $5.2^{\mathrm{a}}$ & $218^{b}$ \\
\hline$C P \quad 170 \ldots \ldots$ & $42.3^{b}$ & $6.72^{b}$ & $.62^{\mathrm{b}}$ & $16.1^{\mathrm{b}}$ & $9.4^{\mathrm{ab}}$ & $7.9^{\mathrm{a}}$ & $226^{b}$ \\
\hline$L G 9 \quad \ldots \ldots \ldots$ & $41.8^{b}$ & $6.66^{b}$ & $.60^{\mathrm{ab}}$ & $16.1^{b}$ & $9.4 \mathrm{ab}$ & $5.1^{\mathrm{a}}$ & $228^{b}$ \\
\hline Rubis 9 ...... & $38.5^{\mathrm{ab}}$ & $6.40^{\mathrm{ab}}$ & $.56^{\mathrm{ab}}$ & $16.7^{\mathrm{e}}$ & $8.9^{\mathrm{a}}$ & $10.0^{\mathrm{b}}$ & $220^{b}$ \\
\hline Prior .......... & $43.1^{b}$ & $6.30^{\mathrm{ab}}$ & $.58^{\mathrm{ab}}$ & $14.6^{\mathrm{a}}$ & $9.5^{\mathrm{ab}}$ & $7.5^{\mathrm{a}}$ & $222^{b}$ \\
\hline$H I T \quad \ldots \ldots \ldots \ldots$ & $39.1 \mathrm{ab}$ & $6.26^{\mathrm{ab}}$ & $.60^{\mathrm{ab}}$ & $16.0^{\mathrm{b}}$ & $9.5^{\mathrm{ab}}$ & $13.3^{\mathrm{bc}}$ & $216^{\mathrm{ab}}$ \\
\hline Hansa ........ & $36.5^{\mathrm{a}}$ & $6.12^{\mathrm{ab}}$ & $.55^{\mathrm{ab}}$ & $17.1^{\mathrm{e}}$ & $9.1^{\mathrm{ab}}$ & $12.5^{b c}$ & $223^{b}$ \\
\hline$L G>\ldots \ldots \ldots$ & $40.1^{b}$ & $6.07^{\mathrm{ab}}$ & $.58^{\mathrm{ab}}$ & $15.1^{\mathrm{ab}}$ & $9.4^{\mathrm{ab}}$ & $8.6^{\mathrm{ab}}$ & $216^{\mathrm{ab}}$ \\
\hline$L G \quad 5 \ldots \ldots \ldots$ & $38.2^{\mathrm{ab}}$ & $6.00^{\mathrm{ab}}$ & $.58^{\mathrm{ab}}$ & $15.8^{\mathrm{b}}$ & $9.8^{\mathrm{b}}$ & $7.1^{\mathrm{a}}$ & $218^{b}$ \\
\hline Flash ........... & $36.7^{\mathrm{a}}$ & $5.80^{\mathrm{a}}$ & $.52^{\mathrm{a}}$ & $15.8^{\mathrm{b}}$ & $9.0^{\mathrm{ab}}$ & $9.7 \mathrm{ab}$ & $214^{\mathrm{a}}$ \\
\hline EDO .......... & $33.8^{\mathrm{a}}$ & $5.54^{\mathrm{a}}$ & $.52^{\mathrm{a}}$ & $16.4^{\mathrm{bc}}$ & $9.5^{\mathrm{ab}}$ & $15.4^{\mathrm{e}}$ & $205^{\mathrm{a}}$ \\
\hline \multirow{2}{*}{$\begin{array}{r}A C G \quad 200 \ldots . \\
\text { Avg. }\end{array}$} & $38.0^{\mathrm{ab}}$ & $5.54^{\mathrm{a}}$ & $.55^{\mathrm{ab}}$ & $14.6^{\mathrm{a}}$ & $9.8^{\mathrm{b}}$ & $9.2^{\mathrm{ab}}$ & $214^{\mathrm{a}}$ \\
\hline & 39.3 & 6.20 & .58 & 15.8 & 9.4 & 9.3 & 218 \\
\hline LSD .05 $\quad \ldots$. & 6.0 & .93 & .09 & .9 & .9 & 4.8 & 12 \\
\hline
\end{tabular}

Table 8. Dry matter yields, dry matter and protein content of twelve corn varieties studied from stens and ears in $1976-78$ in southern Finland.

\begin{tabular}{|c|c|c|c|c|c|c|}
\hline \multirow{2}{*}{ Variety } & \multicolumn{3}{|c|}{ Stems } & \multicolumn{3}{|c|}{ Ears } \\
\hline & DM tons/ha & DM \% & Prot. \% & DM kg/ha & DM \% & Prot. \% \\
\hline LG 11 ................... & $6.48^{b}$ & $18.0^{\mathrm{b}}$ & $7.9^{\mathrm{ab}}$ & $360^{\mathrm{a}}$ & $7.1^{\mathrm{a}}$ & $18.0^{\mathrm{b}}$ \\
\hline 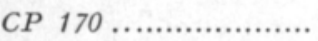 & $6.10^{\mathrm{ab}}$ & $17.4^{\mathrm{b}}$ & $8.2^{\mathrm{ab}}$ & $548^{\mathrm{a}}$ & $7.5^{\mathrm{a}}$ & $16.2^{\mathrm{b}}$ \\
\hline$L G 9 \ldots \ldots \ldots \ldots \ldots \ldots \ldots \ldots \ldots \ldots \ldots \ldots$ & $6.11^{\mathrm{ab}}$ & $16.7^{\mathrm{ab}}$ & $8.7^{b}$ & $316^{\mathrm{a}}$ & $7.5 \mathrm{a}$ & $17.8^{\mathrm{b}}$ \\
\hline Rubis ....................... & $5.84^{\mathrm{ab}}$ & $18.0^{\mathrm{b}}$ & $7.0^{\mathrm{a}}$ & $637^{\mathrm{ab}}$ & $8.5^{\mathrm{ab}}$ & $16.4^{\mathrm{b}}$ \\
\hline 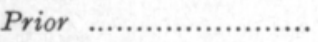 & $5.90^{\mathrm{ab}}$ & $15.6^{\mathrm{a}}$ & $8.8^{\mathrm{b}}$ & $462^{\mathrm{a}}$ & $7.7^{\mathrm{a}}$ & $18.7^{\mathrm{b}}$ \\
\hline 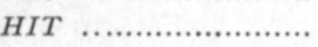 & $5.74^{\mathrm{ab}}$ & $17.6^{\mathrm{b}}$ & $7.6^{\mathrm{ab}}$ & $938^{\mathrm{b}}$ & $10.5^{b}$ & $13.8^{\mathrm{a}}$ \\
\hline Hansa ..................... & $5.13^{\mathrm{ab}}$ & $18.0^{\mathrm{b}}$ & $7.8^{\mathrm{ab}}$ & $693^{\mathrm{ab}}$ & $11.1^{\mathrm{b}}$ & $13.8^{\mathrm{a}}$ \\
\hline$L G>\ldots \ldots \ldots \ldots \ldots \ldots \ldots \ldots \ldots \ldots \ldots$ & $6.11^{\mathrm{ab}}$ & $16.6^{\mathrm{ab}}$ & $8.1^{\mathrm{ab}}$ & $574^{\mathrm{ab}}$ & $8.8^{\mathrm{ab}}$ & $15.2^{\mathrm{ab}}$ \\
\hline$L G 5 \ldots \ldots \ldots \ldots \ldots \ldots \ldots \ldots \ldots \ldots \ldots \ldots$ & $5.84^{\mathrm{ab}}$ & $16.8^{\mathrm{ab}}$ & $8.0^{\mathrm{ab}}$ & $444^{\mathrm{a}}$ & $8.5^{\mathrm{ab}}$ & $16.8^{\mathrm{b}}$ \\
\hline 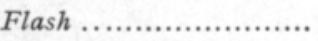 & $5.53^{\mathrm{ab}}$ & $16.9^{\mathrm{ab}}$ & $7.9^{\mathrm{ab}}$ & $625^{\mathrm{ab}}$ & $10.4^{\mathrm{b}}$ & $14.2^{\mathrm{a}}$ \\
\hline 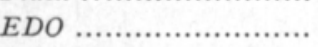 & $5.06^{\mathrm{a}}$ & $18.0^{\mathrm{b}}$ & $8.7^{b}$ & $987^{b}$ & $10.3^{\mathrm{b}}$ & $15.5^{\mathrm{ab}}$ \\
\hline 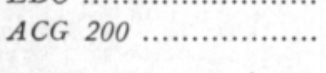 & $5.79 \mathrm{ab}$ & $17.1^{\mathrm{ab}}$ & $7.9^{\mathrm{ab}}$ & $532^{\mathrm{a}}$ & $8.6^{\mathrm{ab}}$ & $16.7^{\mathrm{b}}$ \\
\hline Avg. & 5.80 & 17.2 & 8.1 & 593 & 8.9 & 16.1 \\
\hline LSD .05 .. & . 1.36 & 1.6 & 1.5 & 384 & 2.0 & 1.8 \\
\hline
\end{tabular}

Temperature requirements in the developmental phases of maize

The average time for emergence of all varieties in 1976-78 was 15 days (Table 9). No differences could be found between the emergence times of the varieties. The temperature requirements for emergence, tasseling and silking were counted in three different ways (Table 9). Corn heat units (CHU) appeared to be the most precise measure for emergence. The average day temperature during germination was $11^{\circ} \mathrm{C}$, which is $1.8^{\circ} \mathrm{C}$ lower than that 
Table 9. Average number of days, degree days (DD), effective degree days (EDD) and corn heat units (CHU) of all corn varieties, studied in $1976-78$ in southern Finland.

\begin{tabular}{|c|c|c|c|c|c|}
\hline & & 1976 & 1977 & 1978 & Avg. \\
\hline \multirow[t]{4}{*}{ Emergence } & Days from seeding & 12 & 21 & 11 & 15 \\
\hline & ${ }^{\circ} \mathrm{C}$ DD & 139 & 195 & 149 & 161 \\
\hline & ${ }^{\circ} \mathrm{C}$ EDD & 30 & 15 & 39 & 28 \\
\hline & ${ }^{\circ} \mathrm{C} \mathrm{CHU}$ & 135 & 144 & 143 & 141 \\
\hline \multirow[t]{4}{*}{ Tasseling } & Days from seeding & 93 & 90 & 77 & 89 \\
\hline & ${ }^{\circ} \mathrm{C}$ DD & 1256 & 1213 & 1119 & 1196 \\
\hline & ${ }^{\circ} \mathrm{C}$ EDD & 340 & 347 & 348 & 346 \\
\hline & ${ }^{\circ} \mathrm{C} \mathrm{CHU}$ & 1268 & 1203 & 1133 & 1201 \\
\hline \multirow[t]{4}{*}{ Silking } & Days from seeding & 105 & 100 & 86 & 97 \\
\hline & ${ }^{\circ} \mathrm{C}$ DD & 1411 & 1350 & 1240 & 1334 \\
\hline & ${ }^{\circ} \mathrm{C}$ EDD & 378 & 383 & 386 & 382 \\
\hline & ${ }^{\circ} \mathrm{C} \mathrm{CHU}$ & 1436 & 1344 & 1273 & 1351 \\
\hline
\end{tabular}

recommended for seeding day temperatures in Canada (BROwN 1975). In several tests in Sweden the average time from seeding to emergence has been 14 days, which conforms with the results obtained in Finland (A KERBERG and TORSSELl 1959).

The average growing time from seeding to tasseling during three test years was 89 days. In 1976 the range of all varieties was 85-99 days, the fastest being the variety Velox and the slowest $I$ pho 182 . In 1977 the range was $90-$ 104 days and the extremes were Velox and $L G 9$; in 1978 the range was $71-$ 82 days and the extremes were BC 323 and Silac 233. The results agree with SHAwn's (1955) findings, according to which the time requirement for tasseling is 60 days if the daily average temperature is $21^{\circ} \mathrm{C}$ throughout the period. The effective degree days (EDD) appear to be the most precise means of determining the temperature requirements from seeding to tasseling in 1976-78. The range in EDD during the three years was only $340-348^{\circ} \mathrm{C}$.

Silking of all varieties started on the average 9-12 days after tasseling. The range between the varieties in 1976 was $100-107$ days, in $197796-111$ days, and in 1978 80-90 days. Again EDD seems to be the most precise way of temperature measurement (Table 9).

In variety tests stand height was measured once a week to study the developmental rhythm of silage maize. Plant height and temperature relations were tested with multiple regression. $\mathrm{CHU}$ appears to be the best indicator of plant height development of maize. The other characteristics increased the determination coefficient in the order DD, growing days and EDD. The direct correlation between plant height and temperature was as follows:

$$
\begin{array}{ccc}
\text { Height } \mathrm{cm}-\mathrm{CHU} & \left(\mathrm{r}=.975^{* * *}\right. \\
-\mathrm{EDD} & \left(\mathrm{r}=.970^{* * *}\right. \\
- & -\mathrm{DD} & \left(\mathrm{r}=.967^{* * *}\right. \\
& - \text { Days } & \left(\mathrm{r}=.962^{* * *}\right.
\end{array}
$$


Maize plant height development showed a very distinct sigmoid type growth curve as indicated in Figure 1. The plant height levels represent equal temperature levels in three different heat unit systems. In Figure 2 the dry matter yields of maize are presented in the same way.

\section{Limitations of maize growth and development in Finland}

Maize growth during the Finnish summer is determined by the frost free period and the average temperature of the season. For the Corn Belt area in the USA the ideal season is as follows (WALLACE and BrEssman 1937):

\begin{tabular}{|c|c|}
\hline May: & mean temperature $18{ }^{\circ} \mathrm{C} \quad 85 \mathrm{~mm}$ of rain \\
\hline June: & mean temperature $21.5^{\circ} \mathrm{C}$ \\
\hline July & mean temperature $22.8^{\circ} \mathrm{C} 115 \mathrm{~mm}$ of rain \\
\hline August: & mean temperature $22.8^{\circ} \mathrm{C} 115 \mathrm{~mm}$ of rain \\
\hline
\end{tabular}

Table 10 gives the occurrences of minimum autumn temperatures of 0 and $-2^{\circ} \mathrm{C}$ in four locations in southern Finland. The line connecting these four locations is roughly the northern limit for tentative maize production in this country. The killing frost $-2^{\circ} \mathrm{C}$ usually falls between September 28 and October 16. The $95 \%$ confidence linits of the first fatal frost are between September 16 and October 16. Thus the frost free growing season for maize will roughly be from June 1 to September 16 .

Table 11 gives the distribution of the growing seasons of 16 years into DD-classes at the same four locations. The best early varieties available at present produce a mature grain crop at DD-sums of about 2000 with no interfering late spring or early autumn frosts. This means that one can expect to harvest a mature grain yield twice in ten years.

If the condition for late milk stage silage yield is DD 1900 and the first fatal frost in the later part of September, one may expect a reasonable crop of this kind some six times in ten years in Finland.

Table 10. The occurrence of $\pm 0{ }^{\circ} \mathrm{C}$ and $-2{ }^{\circ} \mathrm{C}$ frost in the autumn and $95 \%$ confidence limits, average 1963-78, four locations in southern Finland.

\begin{tabular}{lrrrr}
\hline Frost limits & Turku & Jokioinen & Tikkurila & Lappeenranta \\
\hline $0^{\circ} \mathrm{C}$ avg. & $22 / 9$ & $11 / 9$ & $17 / 9$ & $27 / 9$ \\
$\geq \mathrm{P} .95 \% \leq$ & $16 / 9-28 / 9$ & $1 / 9-21 / 9$ & $9 / 9-25 / 9$ & $22 / 9-2 / 10$ \\
$\mathrm{~V} \%$ & 71.2 & 65.8 & 117.7 & 51.7 \\
$-2^{\circ} \mathrm{C}$ avg. & $6 / 10$ & $30 / 9$ & $28 / 9$ & $6 / 10$ \\
$\geq \mathrm{P} .95 \% \leq$ & $27 / 9-16 / 10$ & $22 / 9-9 / 10$ & $16 / 9-10 / 10$ & $30 / 9-13 / 10$ \\
$\mathrm{~V} \%$ & 62.1 & 78.5 & 137.8 & 62.5 \\
\hline
\end{tabular}

A satisfactory silage crop can be reached at DD 1800 with no fatal frost earlier than Sept. 20. Along the Finnish coastline (and on the Åland islands) one can expect such conditions eight times in ten years. Although we have 
Table 11. The distribution of growing seasons of years 1963-1978 according to the degree days into six temperature classes in four locations of southern Finland and the average degree days between May $11-$ Sept. 27 of sixteen years.

\begin{tabular}{|c|c|c|c|c|}
\hline \multirow{2}{*}{$\begin{array}{l}\text { Degree } \\
\text { Days }{ }^{\circ} \mathrm{C}\end{array}$} & \multicolumn{4}{|c|}{ Number of years } \\
\hline & Turku & Jokioinen & Tikkurila & Lappeenranta \\
\hline 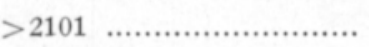 & 1 & - & 2 & 2 \\
\hline 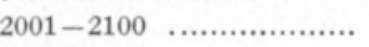 & 3 & 2 & 3 & 2 \\
\hline $1901-2000 \quad \ldots \ldots \ldots \ldots \ldots \ldots \ldots . .$. & 7 & 2 & 5 & 3 \\
\hline $1801-1900 \quad \ldots \ldots \ldots \ldots \ldots \ldots . . . . . . . . .$. & 4 & 7 & 5 & 6 \\
\hline 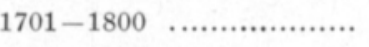 & 1 & 4 & 1 & 3 \\
\hline $1601-1700 \quad \ldots \ldots \ldots \ldots \ldots \ldots \ldots$ & - & 1 & - & - \\
\hline Avg. ${ }^{\circ} \mathrm{C} \quad \ldots \ldots \ldots .$. & 1942 & 1845 & 1952 & 1920 \\
\hline
\end{tabular}

no particular experience of growing maize on the Aland islands it would seem that some areas, where sugarbeets are now grown particularly well, should also be well suited for maize.

Summarizing from Tables 1,10 and 11 one may conclude that maize production in such extremely northerly areas as southern Finland is limited basically due to the occurrence of fatal low temperatures during the vegetative period, which otherwise would be long enough under normal years. This temperature deficit is particularly hazardous at the beginning of the growing season. In particular, resistance to occasional low temperatures should thus, be emphasized in maize breeding programs for extremely northern locations.

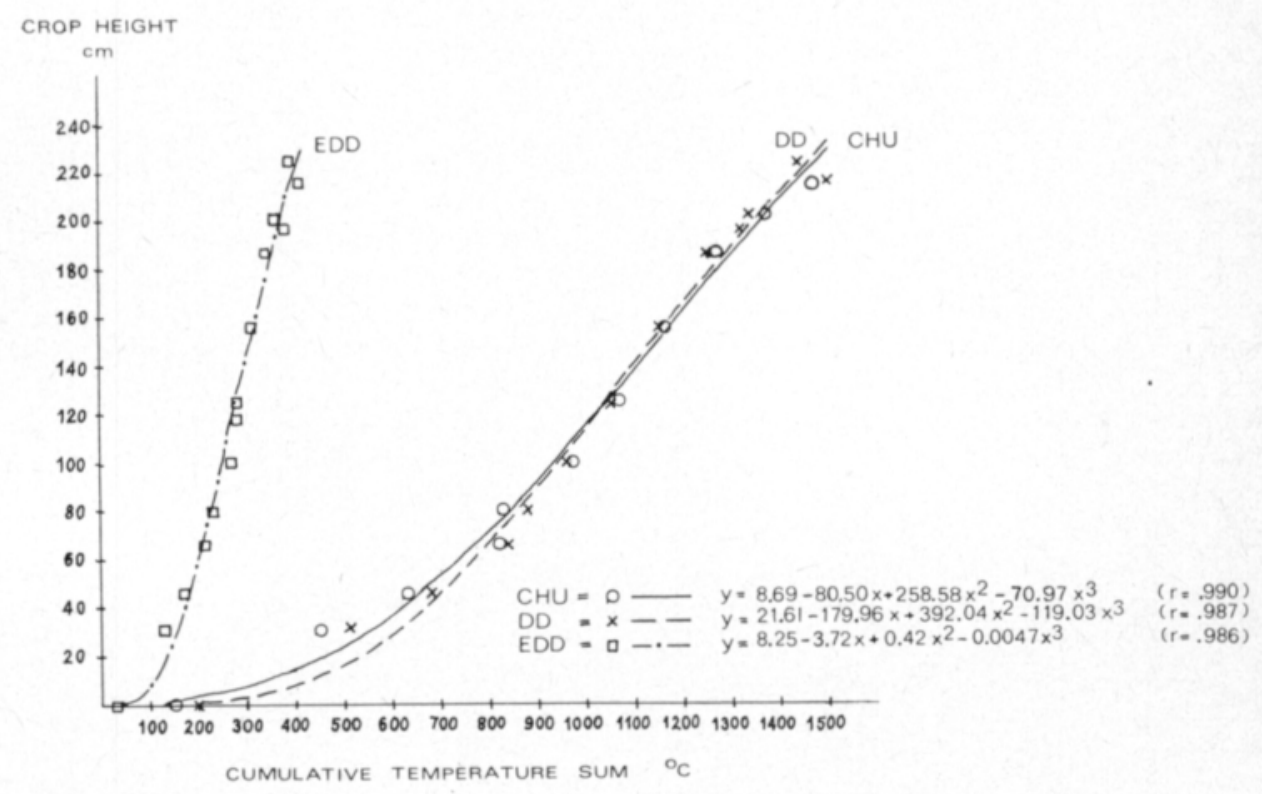

Fig. 1. The relationship between the crop height and cumulative temperature sum ${ }^{\circ} \mathrm{C}$ during the growing season expressed in degree days (DD), in effective degree days (EDD) and in corn heat units (CHU) in variety tests in 1976-78. 


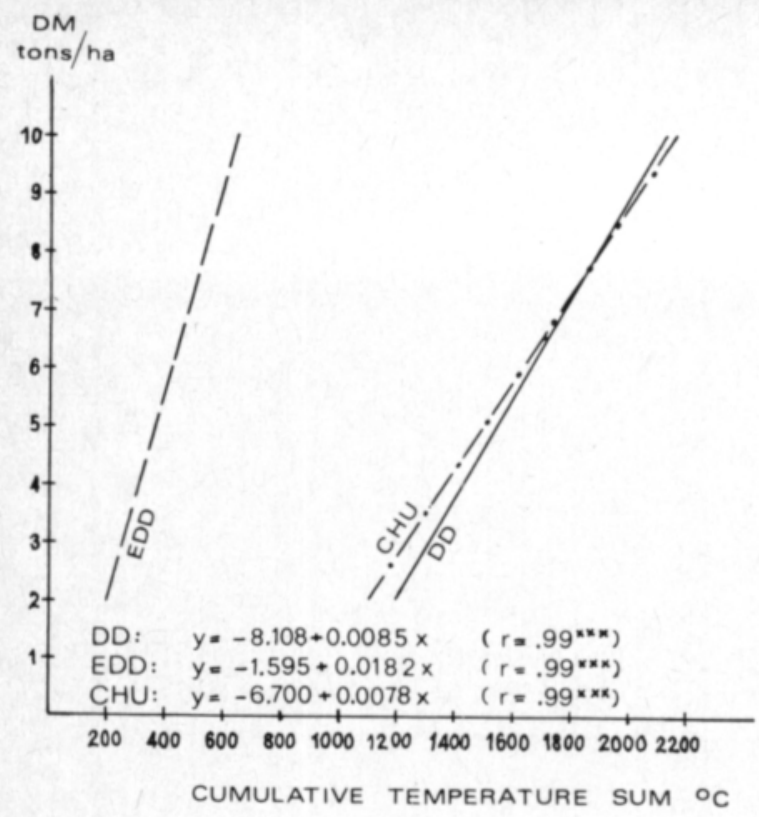

Fig. 2. The relationship between the dry matter production of maize and the cumulative temperature sum ${ }^{\circ} \mathrm{C}$ expressed in degree days (DD), in effective degree days (EDD) and in corn heat units (CHU).

\section{REFERENCES}

ANDERsson, G. \& LööF, G. 1959. Försöks- och förädlingsarbeten med ensilagemajs vid Sveriges Utsädesförening. Växtodling 11: 109-125.

Brown, D. M. 1975. Heat units for corn in Southern Ontario. Ministry of Agriculture and Food. Factsheet N:o 75-077. 4 p.

JuUti, T. \& Raininko, K. 1975. Lisärehukasvit. Hankkijan Siemenjulkaisu 1975: 100-110. NoRDFELT, SAM 1959. Ensilage av grönfodermajs. Växtodling 11:65-76.

Pedersen, K. E. 1975. Sorter af majs til grönhöst 1972-74. Stat. Fors. virks. Pl.kultur. Medd. 1237. 2 p.

RaınıNкo, K. 1970. Lisärehukasvit. Hankkijan Kasvinjalostuslaitos. Siemenjulkaisu 1970: $111-125$.

Ravantti, S. 1956. Rehumaissin viljelemisestä Ruotsissa ja Suomessa. Karjatalous: 98-99.

- 1960. Lisärehukasvit. Hankkijan Kasvinjalostuslaitos. Siemenjulkaisu 1960: 156175.

Shawn, R. 1955. Climatic requirement. Corn and corn improvement. Agronomy. Vol. 5. ed. G. F. Sprague. 315-341. New York: Acad. Press. Inc.

Wallace, H. A. \& Bressman, E. N. 1937. Corn and corn growing. 431 p. John Wiley \& Sons, New York.

VIRTANEN, A. I. 1938. Kokemuksia maissin ja maissi-peluskin viljelyksestä maassamme. Karjatalous: 14: 243-253.

- 1940. Maissin viljelyksestä ensi kesänä. Karjatalous 8: Erip. 7 p.

ÅkerberG, E. \& Torsselle, B. 1959. Förädlingsmetodiska undersökningar med ensilagemajs. Växtodling 11: 65-107.

YLLö, L. 1962. Maissin viljelykokeista Suomessa. Maatal. ja Koetoim. 16: 101-110.

Ms received May 3, 1979. 


\title{
Maissilajikkeiden sopeutumisesta Suomen kasvuoloihin
}

\author{
Seppo Pulli \\ Helsingin yliopisto, Kasvinviljelytieteen laitos, 00710 Helsinki 71 \\ R. M. A. Tigerstedt \\ Helsingin yliopisto, Kasvinjalostustieteen laitos, 00710 Helsinki 71 \\ Osmo Kara \\ Helsingin yliopisto, Maatalousteknologian laitos, 00710 Helsinki 71 \\ G. BRÜNINGHAUS \\ Wiurila, 24910 Halikko
}

Maissin lajikekokeet Suomen Kulttuurirahaston tuella käynnistettiin Wiurilassa, Salossa vuonna 1975. Vuoden 1975 alustavissa kokeissa oli 280 lajiketta. Näistä 19-23 valittiin lopullisiin lajikekokeisiin Yliopiston koetilalle Siuntioon vuosiksi 1976-78. Sääolosuhteet vuonna 1975 olivat keskimääräistä paremmat, vuosina 1976-78 keskimääräistä huomattavasti heikommat.

Kuiva-ainesadot 1975 olivat $5.8-11.5$ tn/ha. Vuosina $1976-78$ vaihtelut kuiva-ainesadoissa heikoimman ja parhaimman lajikkeen välillä olivat 3.8-8.0 tn/ha. Suotuisana kasvukautena 1975 kovan, kypsän siemenen tuotti 44 lajiketta. Vuonna 1976-77 kypsää siementä ei tuottanut yksikään lajike, vuonna 1978 yksi lajike. Säilörehuasteisen, kellertävän tai keltaisen siemenen tuotti vuonna 1977 kahdeksan lajiketta, vuonna 1978 yhdeksän lajiketta ja vuonna 1976 ei yhtään lajiketta.

Lajikekokeissa löydettiin muutamia lupaavia hybridejä Ranskasta, Saksasta ja Jugoslaviasta. Pitkäaikaisiin lämpötilatietoihin perustuen voidaan todeta, että aikaisista hybrideistä saadaan nykyisillä lajikkeilla kasvuoloissamme kypsä jyväsato kahdesti kymmenessä vuodessa, hyvă säilörehusato kuudesti kymmenessä vuodessa ja tyydyttävä säilörehusato kahdeksan kertaa kymmenessä vuodessa.

Maissin kasvun ja kehityksen tärkein rajoittava tekijä kasvuoloissamme on alhainen vuorokautinen lämpötila koko kasvukaudella. Alhaisesta keskilämpötilasta johtuen lämpötilasumma jää useasti alle $1900^{\circ} \mathrm{C}$, jota on pidettävä kannattavan säilörehumaissin viljelyn alarajana. Toinen merkittävä tekijä on ensimmäisen tappavan syyshallan ajoittuminen useasti syyskuun alkupuolelle, mikä oleellisesti vähentää maissin kasvuaikaa. Kevään ja alkukesän usein alhaiset lämpötilat hidastavat maissin alkukehitystä. Tästä syystä maissilla nopean kasvun vaihe saavutetaan vasta elokuun vaihteessa, jolloin lämpöoloissa, ja varsinkin säteilymäärissä on tapahtunut merkittävä vähentyminen. 